\title{
Scattering State of Klein-Gordon Particles by q-Parameter Hyperbolic Poschl-Teller Potential
}

\author{
Akpan Ndem Ikot, ${ }^{1}$ Hillary P. Obong, ${ }^{1}$ Israel O. Owate, ${ }^{1}$ \\ Michael C. Onyeaju, ${ }^{1}$ and Hassan Hassanabadi ${ }^{2}$ \\ ${ }^{1}$ Theoretical Physics Group, Department of Physics, University of Port Harcourt, PMB 5323, Choba, Port Harcourt, Nigeria \\ ${ }^{2}$ Physics Department, University of Shahrood, Shahrood, Iran
}

Correspondence should be addressed to Akpan Ndem Ikot; ndemikotphysics@gmail.com

Received 2 June 2015; Revised 14 July 2015; Accepted 16 July 2015

Academic Editor: Reinhard Schlickeiser

Copyright (C) 2015 Akpan Ndem Ikot et al. This is an open access article distributed under the Creative Commons Attribution License, which permits unrestricted use, distribution, and reproduction in any medium, provided the original work is properly cited. The publication of this article was funded by SCOAP S $^{3}$

\begin{abstract}
The one-dimensional Klein-Gordon equation for equal vector and scalar q-parameter hyperbolic Poschl-Teller potential is solved in terms of the hypergeometric functions. We calculate in detail the solutions of the scattering and bound states. By virtue of the conditions of equation of continuity of the wave functions, we obtained explicit expressions for the reflection and transmission coefficients and energy equation for the bound state solutions.
\end{abstract}

\section{Introduction}

The scattering states of the relativistic and nonrelativistic wave equation in recent times have received great attention in physics [1-9]. Scattering and bound state solutions of asymmetric Hulthen potential have been obtained by Arda and Sever [10]. Also, the bound state and scattering state of Klein-Gordon equation with effective mass formalism have been studied by Aydoğdu et al. [11]. Aydoğdu et al. [12] examined the scattering and bound states of massive Dirac equation with Woods-Saxon potential. Rojas and Vilalba [13] in their investigation had shown the relation between the bound state energy eigenvalues and transmission resonances for the Klein-Gordon particle with Woods-Saxon potential as the same for the Dirac particle. Hassanabadi et al. [14, 15] investigated the scattering state of relativistic spinless particles with Kink-like potential and scattering of Woods-Saxon potential in the framework of minimal length. The scattering states of relativistic and nonrelativistic particles with WoodsSaxon potential and mass dependent Woods-Saxon potential have been studied by Alpdoğan et al. [16] and Arda et al. [17]. Recently, Alpdoğan studied the scattering of massive Dirac particles with generalized asymmetric Woods-Saxon potential [18]. Yanar et al. studied the scattering and bound state of Duffin-Kemmer-Petiau particles with q-parameter hyperbolic Poschl-Teller (qHPT) potential [19]. The qHPT potential is defined as follows:

$$
\begin{aligned}
& V(x)=\frac{4 \lambda(\lambda-1)}{\cosh _{q}^{2}(\alpha x)}=4 \lambda(\lambda-1) \\
& \cdot\left[\theta(-x) \frac{e^{2 \alpha x}}{\left(1+q e^{2 \alpha x}\right)^{2}}+\theta(x) \frac{e^{-2 \alpha x}}{\left(1+q e^{-2 \alpha x}\right)^{2}}\right],
\end{aligned}
$$

where $\theta(x)$ is the step function, $q$ is the deformation parameter and $q \neq 0, \lambda$ is the height of the potential, $\lambda \neq 0, \lambda \neq 1$, and $\alpha$ is the range of the potential barrier. This potential plays an important role in describing the interactions in molecular, atomic, and nuclear physics. One of the findings of physics is to understand the structures of nucleus, atoms, molecules, and other material objects. Thus, the sole aim of physicists is to create unique models that contain the physically motivated potential that will describe the interactions between particles. A few numbers of these potential models have been identified to describe the interaction in the nuclei and diatomic and polyatomic molecules [20, 21]. The applications of the PoschlTeller-like type potential for analyzing the bound states energy of $\Lambda$-particle in hypernuclei in nuclear physics had 
been reported [19]. Furthermore, the qHPT potential may found much usefulness in molecular, atomic, and nuclear physics.

Understanding of our knowledge about fine scale systems has been gained by investigations into scattering and bounded state of such systems. Therefore, the scattering problem has become an interesting topic in relativistic or nonrelativistic quantum mechanics. In the case of nonrelativistic scattering problem, it has been shown that transmission and reflection coefficients take 1 and 0 , respectively, as external potential has well behaved at infinity for the zero energy limit [22-24]. However, reflection coefficient goes to zero while transmission coefficient goes to unity in the zero energy limit when external potential supports a half-bound state. This situation was called as transmission resonance [25] which is escorted by fluctuations phenomena. The transmission resonance concept has been recently generalized to the relativistic case [26].

In this work, we attempt to study the solution of scattering state of the Klein-Gordon equation with equal vector and scalar (qHPT) potential. Our aim will be to calculate in detail the reflection $(R)$ and transmission $(T)$ coefficients and obtain the bound state solution of the qHPT potential using the equation of continuity of the wave function.

\section{Scattering State Solutions of Klein-Gordon Equation for the qHPT Potential}

The time-independent Klein-Gordon equation with equal scalar $S(x)$ and vector $V(x)$ potentials can be written as [12]

$$
\begin{aligned}
\frac{d^{2} \psi(x)}{d x^{2}}+\left\{E^{2}-m^{2}-2(E+m) V(x)\right\} \psi(x) & =0, \\
& (\hbar=c=1),
\end{aligned}
$$

where $E$ is the relativistic energy of the particles and $m$ is the mass of the particle. In order to obtain the scattering solution for qHPT potential, we consider both $x<0$ and $x>0$ at $|E|>m$. We intend to study the scattering of (2); we now seek the wave function for the case $x<0$. By substituting (1) into (2), we have

$$
\begin{aligned}
& \frac{d^{2} \psi(x)}{d x^{2}} \\
& \quad+\left\{E^{2}-m^{2}-\frac{8 \lambda(\lambda-1)(E+m) e^{2 \alpha x}}{\left(1+q e^{2 \alpha x}\right)^{2}}\right\} \psi(x) \\
& \quad 0 .
\end{aligned}
$$

To solve (3), we used a new variable defined as $z=-q e^{2 \alpha x}$ which yields

$$
\begin{aligned}
& z(1-z) \frac{d^{2} \psi}{d z^{2}}+(1-z) \frac{d \psi}{d z} \\
& +\frac{1}{z(1-z)}\left\{\gamma_{1} z^{2}+\gamma_{2} z+\gamma\right\} \psi(z)=0,
\end{aligned}
$$

where

$$
\begin{aligned}
& \gamma_{1}=\frac{1}{4 \alpha^{2}}\left(E^{2}-m^{2}\right), \\
& \gamma_{2}=\frac{2}{\alpha^{2}}\left(\frac{(E+m) \lambda(\lambda-1)}{q}+\frac{m^{2}-E^{2}}{4}\right), \\
& \gamma_{3}=\frac{1}{4 \alpha^{2}}\left(E^{2}-m^{2}\right) .
\end{aligned}
$$

Defining the wave function in (4) as $\psi(z)=z^{\mu}(1-z)^{\nu} \varphi(z)$, then (4) turns into the hypergeometric differential equation [27]

$$
\begin{gathered}
z(1-z) \frac{d^{2} \varphi}{d z^{2}}+[1+2 \mu-(1+2 \mu+2 \nu) z] \frac{d \varphi}{d z} \\
-(\mu+\nu+\delta)(\mu+\nu-\delta) \varphi(z)=0
\end{gathered}
$$

where $\mu, \nu$, and $\delta$ are defined as follows:

$$
\begin{aligned}
\mu & =\frac{i k}{2 \alpha}, \\
\nu & =\frac{1}{2} \pm \frac{1}{2} \sqrt{1-\frac{8(E+m) \lambda(\lambda-1)}{\alpha^{2} q}}, \\
\delta & =\frac{i k}{2 \alpha}, \\
k^{2} & =\left(E^{2}-m^{2}\right) .
\end{aligned}
$$

Regarding properties of hypergeometric functions the minus sign should be chosen for $\nu$. The solutions of (6) can be written in terms of the hypergeometric function as follows:

$$
\begin{aligned}
\varphi(z<0) \\
=A_{2} F_{1}(\mu+\nu+\delta, \mu+\nu-\delta, 1+2 \mu, z) \\
\quad+B_{2} z^{-2 \mu} F_{1}(-\mu+\nu+\delta,-\mu+\nu-\delta, 1-2 \mu, z) .
\end{aligned}
$$

The general solutions for $x<0$ are given as

$$
\begin{aligned}
& \psi(z<0)=A z^{\mu}(1-z)^{\nu} \\
& \quad \cdot{ }_{2} F_{1}(\mu+\nu+\delta, \mu+\nu-\delta, 1+2 \mu, z) \\
& +B z^{-\mu}(1-z)^{\nu} \\
& \quad \cdot{ }_{2} F_{1}(-\mu+\nu+\delta,-\mu+\nu-\delta, 1-2 \mu, z) .
\end{aligned}
$$

Next, we investigate the solution for $x>0$. Again substituting (1) into (2) yields

$$
\begin{aligned}
& \frac{d^{2} \psi(x)}{d x^{2}} \\
& \quad+\left\{E^{2}-m^{2}-\frac{8 \lambda(\lambda-1)(E+m) e^{-2 \alpha x}}{\left(1+q e^{-2 \alpha x}\right)^{2}}\right\} \psi(x) \\
& \quad 0 .
\end{aligned}
$$


Defining the new variable $\widetilde{z}=-q e^{-2 \alpha x}$ and redefining the ansatz for the wave function as $\psi(\widetilde{z})=\widetilde{z}^{\widetilde{\mu}}(1-\widetilde{z})^{\tilde{\nu}} f(\widetilde{z})$, the solutions of (10) become

$$
\begin{aligned}
& \psi(\widetilde{z}>0)=C \widetilde{z}^{\widetilde{\mu}}(1-\widetilde{z})^{\tilde{\nu}} \\
& \cdot{ }_{2} F_{1}(\widetilde{\mu}+\widetilde{\nu}+\widetilde{\delta}, \widetilde{\mu}+\widetilde{\nu}-\widetilde{\delta}, 1+2 \widetilde{\mu}, \widetilde{z}) \\
& +D \widetilde{z}^{-\widetilde{\mu}}(1-\widetilde{z})^{\widetilde{\nu}} \\
& \quad \cdot{ }_{2} F_{1}(-\widetilde{\mu}+\widetilde{\nu}+\widetilde{\delta},-\widetilde{\mu}+\widetilde{\nu}-\widetilde{\delta}, 1-2 \widetilde{\mu}, \widetilde{z}),
\end{aligned}
$$

where

$$
\begin{aligned}
\widetilde{\mu} & =\frac{i k}{2 \alpha}, \\
\widetilde{v} & =\frac{1}{2} \pm \frac{1}{2} \sqrt{1-\frac{8(E+m) \lambda(\lambda-1)}{q \alpha^{2}}}, \\
\widetilde{\delta} & =\frac{i k}{2 \hbar}, \\
k^{2} & =\left(E^{2}-m^{2}\right) .
\end{aligned}
$$

Here we also should choose the minus sign for $\widetilde{v}$. We seek for the physical results of the problem under investigation; therefore in order to get this physical result, the solutions obtained have to be used with appropriate boundary conditions as $x \rightarrow-\infty$ and $x \rightarrow+\infty$. Applying the asymptotic solution to (9) for $x \rightarrow-\infty$, as $z_{L} \rightarrow 0$ and $(1-z)^{\nu} \rightarrow 1$, becomes

$$
\begin{aligned}
\psi_{L}(x \longrightarrow-\infty)= & A\left(-\frac{1}{q}\right)^{i k / 2 \alpha} e^{i k} \\
& +B\left(-\frac{1}{q}\right)^{-i k / 2 \alpha} e^{-i k} .
\end{aligned}
$$

In order to find a plane wave traveling from left to right, we set $C=0$ in (11) and the asymptotic behavior of the right solution becomes

$$
\psi_{R}(x \longrightarrow \infty)=D(-q)^{-i k / 2 \alpha \hbar c} e^{i k / \hbar c} .
$$

Now in order to give explicit expressions for the coefficients, we use the continuity conditions of the wave function and its derivative defined as

$$
\begin{aligned}
& \psi_{L}(x=0)=\psi_{R}(x=0), \\
& \psi_{L}^{\prime}(x=0)=\psi_{R}^{\prime}(x=0),
\end{aligned}
$$

where the prime denotes differential with respect to $x$. Applying these conditions on the wave function and matching the wave function at $x=0$, we get

$$
\begin{aligned}
& A Q_{1} U_{1}+B Q_{2} U_{2}=D Q_{3} U_{3} \\
& A\left(Q_{4} U_{4}+Q_{5} U_{5}+Q_{6} S_{1} U_{6}\right) \\
& \quad+B\left(Q_{7} U_{7}+Q_{8} U_{8}+Q_{9} S_{2} U_{9}\right) \\
& \quad=D\left(Q_{10} U_{10}+Q_{11} U_{11}+Q_{12} S_{3} U_{12}\right),
\end{aligned}
$$

where the following abbreviations have been used:

$$
\begin{aligned}
& Q_{1}=(-q)^{\mu}(1+q)^{\nu}, \\
& Q_{2}=(-q)^{-\mu}(1+q)^{\nu} \text {, } \\
& Q_{3}=(-q)^{-\widetilde{\mu}}(1+q)^{\tilde{\nu}} \text {, } \\
& Q_{4}=-2 \alpha \mu(-q)^{\mu}(1+q)^{\nu} \text {, } \\
& Q_{5}=2 \alpha \nu(-q)^{\mu+1}(1+q)^{\nu-1} \text {, } \\
& Q_{6}=-2 \alpha(-q)^{\mu+1}(1+q)^{\nu} \text {, } \\
& Q_{7}=2 \alpha \mu(-q)^{-\mu}(1+q)^{\nu} \text {, } \\
& Q_{8}=2 \alpha \nu(-q)^{-\mu+1}(1+q)^{\nu-1} \text {, } \\
& Q_{9}=-2 \alpha(-q)^{-\mu+1}(1+q)^{\nu} \text {, } \\
& Q_{10}=-2 \alpha \widetilde{\mu}(-q)^{-\widetilde{\mu}}(1+q)^{\tilde{\nu}}, \\
& Q_{11}=-2 \alpha \widetilde{\nu}(-q)^{-\widetilde{\mu}+1}(1+q)^{\tilde{\nu}-1}, \\
& Q_{12}=2 \alpha(-q)^{-\tilde{\mu}+1}(1+q)^{\tilde{\nu}} \text {, } \\
& S_{1}=\frac{(\mu+\nu+\delta)(\mu+\nu-\delta)}{(1+2 \mu)}, \\
& S_{2}=\frac{(-\mu+\nu+\delta)(-\mu+\nu-\delta)}{(1-2 \mu)} \text {, } \\
& S_{3}=\frac{(-\widetilde{\mu}+\nu+\delta)(-\widetilde{\mu}+\nu-\delta)}{(1-2 \widetilde{\mu})} \text {, } \\
& U_{1}={ }_{2} F_{1}(\mu+\nu+\delta, \mu+\nu-\delta, 1+2 \mu,-q) \text {, } \\
& U_{2}={ }_{2} F_{1}(-\mu+\nu+\delta,-\mu+\nu-\delta, 1-2 \mu,-q) \text {, } \\
& U_{3}={ }_{2} F_{1}(-\widetilde{\mu}+\widetilde{\nu}+\widetilde{\delta}, \widetilde{\mu}+\widetilde{\nu}-\widetilde{\delta}, 1-2 \widetilde{\mu},-q), \\
& U_{4}={ }_{2} F_{1}(\mu+\nu+\delta, \mu+\nu-\delta, 1+2 \mu,-q) \text {, } \\
& U_{5}={ }_{2} F_{1}(\mu+\nu+\delta, \mu+\nu-\delta ; 1+2 \mu,-q) \text {, } \\
& U_{6}={ }_{2} F_{1}(\mu+\nu+\delta+1, \mu+\nu-\delta+1 ; 2+2 \mu,-q) \text {, } \\
& U_{7}={ }_{2} F_{1}(-\mu+\nu+\delta,-\mu+\nu-\delta, 1-2 \mu,-q), \\
& U_{8}={ }_{2} F_{1}(-\mu+\nu+\delta,-\mu+\nu-\delta, 1-2 \mu,-q) \text {, } \\
& U_{9} \\
& ={ }_{2} F_{1}(-\mu+\nu+\delta+1,-\mu+\nu-\delta+1,2-2 \mu,-q), \\
& U_{10}={ }_{2} F_{1}(-\widetilde{\mu}+\widetilde{\nu}+\widetilde{\delta},-\widetilde{\mu}+\widetilde{\nu}-\widetilde{\delta}, 1-2 \widetilde{\mu},-q) \text {, } \\
& U_{11}={ }_{2} F_{1}(-\widetilde{\mu}+\widetilde{\nu}+\widetilde{\delta},-\widetilde{\mu}+\widetilde{\nu}-\widetilde{\delta}, 1-2 \widetilde{\mu},-q), \\
& U_{12} \\
& ={ }_{2} F_{1}(-\widetilde{\mu}+\widetilde{\nu}+\widetilde{\delta}+1,-\widetilde{\mu}+\widetilde{\nu}-\widetilde{\delta}+1,2-2 \widetilde{\mu},-q) .
\end{aligned}
$$


And the property of hypergeometric function $(d / d s)_{2} F_{1}(a$, $b, c, s)=(a b / c){ }_{2} F_{1}(a+1, b+1, c+1, s)[28]$ has been used in obtaining (17). The one-dimensional current density for the Klein-Gordon equation in terms of the relativistic units $(\hbar=$ $c=1$ ) is given by [29]

$$
J(x)=\frac{i}{2 m}\left(\psi^{*} \nabla_{x} \psi-\psi \nabla_{x} \psi^{*}\right)
$$

The reflection and transmission coefficients are defined in terms of current density as follows:

$$
\begin{aligned}
& R=\frac{j_{\text {ref }}}{j_{\text {inc }}}=\left|\frac{B}{A}\right|^{2}, \\
& T=\frac{j_{\text {trans }}}{j_{\text {inc }}}=\left|\frac{D}{A}\right|^{2} .
\end{aligned}
$$

Now using these equations and after a tedious algebra, we obtain the reflection and transmission coefficients as

$$
\begin{aligned}
& R=\left|\frac{B}{A}\right|^{2}=\left|\frac{Q_{4} U_{4}+Q_{5} U_{5}+Q_{6} S_{1} U_{6}-\left(Q_{1} U_{1} / Q_{3} U_{3}\right)\left(Q_{10} U_{10}+Q_{11} U_{11}+Q_{12} S_{3} U_{12}\right)}{\left(Q_{2} U_{2} / Q_{3} U_{3}\right)\left(U_{10} Q_{10}+Q_{11} U_{11}+Q_{12} S_{3} U_{12}\right)-\left(Q_{7} U_{7}+Q_{8} U_{8}+Q_{9} S_{2} U_{9}\right)}\right|^{2}, \\
& T=\left|\frac{D}{A}\right|^{2}=\left|\frac{\left(Q_{2} U_{2} / Q_{3} U_{3}\right)\left(Q_{4} U_{4}+Q_{5} U_{5}+Q_{6} S_{1} U_{6}\right)-\left(Q_{1} U_{1} / Q_{3} U_{3}\right)\left(Q_{7} U_{7}+Q_{8} U_{8}+Q_{9} S_{2} U_{9}\right)}{\left(Q_{2} U_{2} / Q_{3} U_{3}\right)\left(U_{10} Q_{10}+Q_{11} U_{11}+Q_{12} S_{3} U_{12}\right)-\left(Q_{7} U_{7}+Q_{8} U_{8}+Q_{9} S_{2} U_{9}\right)}\right|^{2} .
\end{aligned}
$$

We have depicted in Figure 5 that the sum of reflection and transmission coefficients approaches unity.

\section{Bound State Solutions of Klein-Gordon Equation for the qHPT Potential}

In order to find the bound state solutions for the KleinGordon particle with qHPT potential, we map $8 \lambda(\lambda-1) \rightarrow$ $-V_{0}$.

3.1. Bound State Solutions in the Negative Region $(x<0)$. The bound state solutions can be found in this region by changing the variable $z=-q e^{2 \alpha x}$ and taking into consideration $8 \lambda(\lambda-$ 1) $\rightarrow-V_{0}$ and (3) becomes

$$
\begin{aligned}
& z(1-z) \frac{d^{2} \psi}{d z^{2}}+(1-z) \frac{d \psi}{d z} \\
& +\frac{1}{z(1-z)}\left\{\beta_{1} z^{2}+\beta_{2} z+\beta\right\} \psi(z)=0,
\end{aligned}
$$

where

$$
\begin{aligned}
& \beta_{1}=\frac{1}{4 \alpha^{2}}\left(E^{2}-m^{2}\right), \\
& \beta_{2}=-\frac{1}{2 \alpha^{2}}\left(\frac{V_{0}(E+m)}{q}+\left(E^{2}-m^{2}\right)\right), \\
& \beta_{3}=\frac{1}{4 \alpha^{2}}\left(E^{2}-m^{2}\right) .
\end{aligned}
$$

Taking a wave function of the form $\psi(z)=z^{\mu_{1}}(1-z)^{\nu_{1}} \varphi(z)$, then (22) turns into the hypergeometric differential equation [27]

$$
\begin{gathered}
z(1-z) \frac{d^{2} \varphi}{d z^{2}}+\left[1+2 \mu_{1}-\left(1+2 \mu_{1}+2 v_{1}\right) z\right] \frac{d \varphi}{d z} \\
-\left(\mu_{1}+v_{1}+\delta_{1}\right)\left(\mu_{1}+v_{1}-\delta_{1}\right) \varphi(z)=0
\end{gathered}
$$

with $\mu_{1}, \nu_{1}$, and $\delta_{1}$ defined as follows:

$$
\begin{aligned}
& \mu_{1}=\frac{i k}{2 \alpha}, \\
& \nu_{1}=\frac{1}{2} \pm \frac{1}{2} \sqrt{1+\frac{(E+m) V_{0}}{\alpha^{2} q}} \\
& \delta_{1}=\frac{i k}{2 \alpha} \\
& k^{2}=\left(E^{2}-m^{2}\right) .
\end{aligned}
$$

We choose minus sign for $v_{1}$ in equation (25). The general solutions for $x<0$ are given as

$$
\begin{aligned}
& \psi(z<0)=A_{1} z^{\mu_{1}}(1-z)^{\nu_{1}} \\
& \quad \cdot{ }_{2} F_{1}\left(\mu_{1}+\nu_{1}+\delta_{1}, \mu_{1}+\nu_{1}-\delta_{1}, 1+2 \mu_{1}, z\right) \\
& +B_{1} z^{-\mu_{1}}(1-z)^{\nu_{1}} \\
& \quad \cdot{ }_{2} F_{1}\left(-\mu_{1}+\nu_{1}+\delta_{1},-\mu_{1}+\nu_{1}-\delta_{1}, 1-2 \mu_{1}, z\right) .
\end{aligned}
$$

3.2. Bound State Solutions in the Positive Region $(x>0)$. In the positive region, we defined the variable $\widetilde{z}=-q e^{-2 \alpha x}$ with $8 \lambda(\lambda-1) \rightarrow-V_{0}$ and taking the wave function $\psi(\widetilde{z})=$ $\widetilde{z}^{\widetilde{\mu}_{1}}(1-\widetilde{z})^{\widetilde{\nu}_{1}} \varphi(\widetilde{z})$ and following the same procedures as the case of negative region, we obtain the solutions for the positive region as follows:

$$
\begin{aligned}
& \psi(\widetilde{z}>0)=C_{1} \widetilde{z}^{\widetilde{\mu}_{1}}(1-\widetilde{z})^{\tilde{\nu}_{1}} \\
& \quad \cdot{ }_{2} F_{1}\left(\widetilde{\mu}_{1}+\widetilde{\nu}_{1}+\widetilde{\delta}_{1}, \widetilde{\mu}_{1}+\widetilde{\nu}_{1}-\widetilde{\delta}_{1}, 1+2 \widetilde{\mu}_{1}, \widetilde{z}\right) \\
& \quad+D_{1} z^{-\widetilde{\mu}_{1}}(1-\widetilde{z})^{\widetilde{\nu}_{1}} \\
& \quad \cdot{ }_{2} F_{1}\left(-\widetilde{\mu}_{1}+\widetilde{\nu}_{1}+\widetilde{\delta}_{1},-\widetilde{\mu}_{1}+\widetilde{\nu}_{1}-\widetilde{\delta}_{1}, 1-2 \widetilde{\mu}_{1}, \widetilde{z}\right),
\end{aligned}
$$


where

$$
\begin{aligned}
& \widetilde{\mu}_{1}=\frac{i k}{2 \alpha}, \\
& \widetilde{\nu}_{1}=\frac{1}{2} \pm \frac{1}{2} \sqrt{1+\frac{2\left(E+m c^{2}\right) V_{0}}{\alpha^{2} q}} \\
& \widetilde{\delta}_{1}=\frac{i k}{2 \alpha} \\
& k^{2}=\left(E^{2}-m^{2}\right) .
\end{aligned}
$$

And as mentioned above the minus sing should be chosen. In order to find the equation for the energy eigenvalues, we set $B=D=0$ and used the conditions of continuity for the wave function as $\psi_{L}(x=0)=\psi_{R}(x=0), \psi_{L}^{\prime}(x=0)=\psi_{R}^{\prime}(x=0)$ and we get

$$
\begin{aligned}
& A_{1} a_{1} K_{1}=C_{1} b_{1} K_{2}, \\
& A_{1}\left(a_{2} K_{3}+a_{3} K_{4}+a_{5} K_{5}\right) \\
& \quad=C_{1}\left(b_{2} K_{6}+b_{3} K_{7}+b_{4} K_{8}\right),
\end{aligned}
$$

where

$$
\begin{aligned}
& a_{1}=(-q)^{\mu_{1}}(1+q)^{\nu_{1}} \text {, } \\
& K_{1}={ }_{2} F_{1}\left(\mu_{1}+\nu_{1}+\delta_{1}, \mu_{1}+\nu_{1}-\delta_{1}, 1+2 \mu_{1} ;-q\right) \text {, } \\
& b_{1}=(-q)^{\widetilde{\mu}_{1}}(1+q)^{\widetilde{\nu}_{1}} \text {, } \\
& K_{2}={ }_{2} F_{1}\left(\widetilde{\mu}_{1}+\widetilde{\nu}_{1}+\widetilde{\delta}_{1}, \widetilde{\mu}_{1}+\widetilde{\nu}_{1}-\widetilde{\delta}_{1}, 1+2 \widetilde{\mu}_{1} ;-q\right) \text {, } \\
& a_{2}=2 \alpha \mu_{1}(-q)^{\mu_{1}}(1+q)^{\nu_{1}} \text {, } \\
& a_{3}=2 \alpha \nu(-q)^{\mu_{1}+1}(1+q)^{\nu_{1}-1} \text {, } \\
& a_{4}=2 \alpha(-q)^{\mu_{1}+1}(1+q)^{\nu_{1}} \text {, } \\
& K_{3}={ }_{2} F_{1}\left(\mu_{1}+\nu_{1}+\delta_{1}, \mu_{1}+\nu_{1}-\delta_{1}, 1+2 \mu_{1} ;-q\right) \text {, } \\
& K_{4}={ }_{2} F_{1}\left(\mu_{1}+\nu_{1}+\delta_{1}, \mu_{1}+v_{1}-\delta_{1}, 1+2 \mu_{1} ;-q\right) \text {, } \\
& b_{2}=-2 \alpha \widetilde{\mu}_{1}(-q)^{\tilde{\mu}_{1}}(1+q)^{\tilde{\nu}_{1}} \text {, } \\
& b_{3}=2 \alpha \widetilde{\nu}_{1}(-q)^{\widetilde{\mu}_{1}+1}(1+q)^{\tilde{\nu}_{1}-1} \text {, } \\
& b_{4}=-2 \alpha(-q)^{\widetilde{\mu}_{1}+1}(1+q)^{\widetilde{\nu}_{1}} \text {, } \\
& K_{5}=\frac{\left(\mu_{1}+\nu_{1}+\delta_{1}\right)\left(\mu_{1}+\nu_{1}-\delta_{1}\right)}{1+2 \mu_{1}}{ }_{2} F_{1}\left(\mu_{1}+\nu_{1}+\delta_{1}\right. \\
& \left.+1, \mu_{1}+\nu_{1}-\delta_{1}+1,2+2 \mu_{1} ;-q\right) \text {, } \\
& K_{6}={ }_{2} F_{1}\left(\tilde{\mu}_{1}+\widetilde{\nu}_{1}+\widetilde{\delta}_{1}, \tilde{\mu}_{1}+\widetilde{\nu}_{1}-\widetilde{\delta}_{1}, 1+2 \tilde{\mu}_{1} ;-q\right) \text {, } \\
& K_{7}={ }_{2} F_{1}\left(\widetilde{\mu}_{1}+\widetilde{\nu}_{1}+\widetilde{\delta}_{1}, \widetilde{\mu}_{1}+\widetilde{\nu}_{1}-\widetilde{\delta}_{1}, 1+2 \widetilde{\mu}_{1} ;-q\right) \text {, }
\end{aligned}
$$

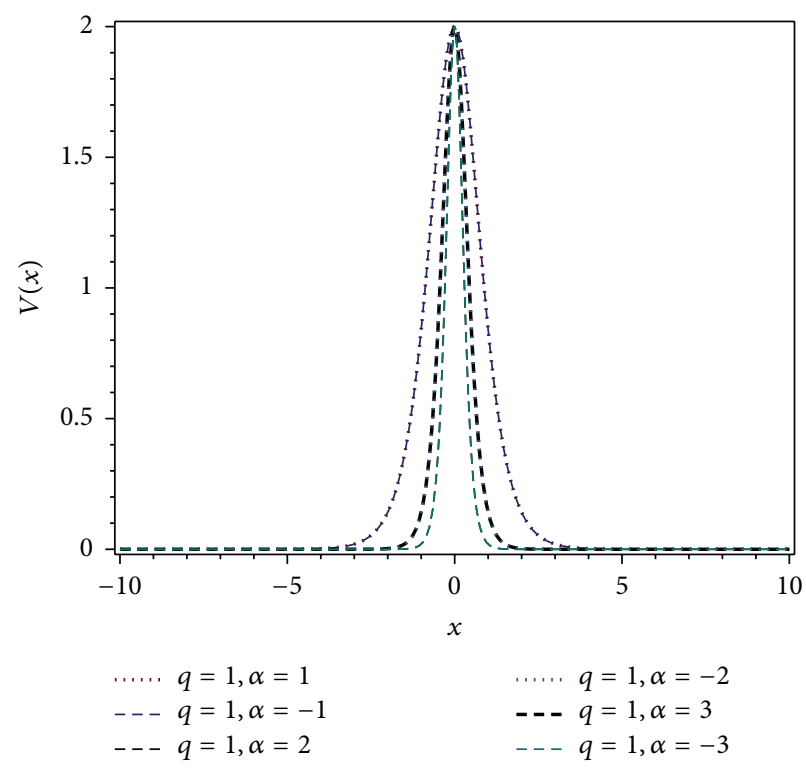

FIGURE 1: The plot of the qHPT potential as a function of position with $q=1, \alpha=1,2$, and 3 for $x<0$ and $x>0$, respectively.

$$
\begin{aligned}
K_{8} & =\frac{\left(\widetilde{\mu}_{1}+\widetilde{\nu}_{1}+\widetilde{\delta}_{1}\right)\left(\widetilde{\mu}_{1}+\widetilde{\nu}_{1}-\widetilde{\delta}_{1}\right)}{1+2 \widetilde{\mu}_{1}}{ }_{2} F_{1}\left(\widetilde{\mu}_{1}+\widetilde{\nu}_{1}+\widetilde{\delta}_{1}\right. \\
& \left.+1, \widetilde{\mu}_{1}+\widetilde{\nu}_{1}-\widetilde{\delta}_{1}+1,2+2 \widetilde{\mu}_{1} ;-q\right) .
\end{aligned}
$$

Equation (29) admits a solution if and only if its determinant is zero [30]. This thus provides a condition for getting the energy eigenvalues as

$$
\begin{aligned}
& b_{1} a_{2} K_{2} K_{3}+b_{1} a_{3} K_{2} K_{4}+b_{1} a_{4} K_{2} K_{5} \\
& \quad=a_{1} b_{2} K_{1} K_{6}+a_{1} b_{3} K_{1} K_{7}+a_{1} b_{4} K_{1} K_{8} .
\end{aligned}
$$

Energy equation (31) is a complicated transcendental equation and can only be solved numerically.

\section{Results and Discussion}

The behaviors of the qHPT potential as a function of $x$ are displayed in Figures 1-4 for various values of qHPT potential parameters. For $q=0$ and $8 \lambda(\lambda-1) \rightarrow V_{0}$, the qHPT potential turns to cusp potential which is the consistent one reported in [31]. However, if we map $E-M=2 \mu / \hbar^{2}$ and $E+$ $M=E_{n l}$ in (31) our results reduced to the cusp potential for the nonrelativistic regime as reported in [32].

\section{Conclusions}

We solved the relativistic spinless Klein-Gordon particles for the qHPT potential and calculated the wave functions that describe the scattering states in terms of hypergeometric function. By mapping of the potential parameter of qHPT potential, we also solved the bound state solution of the KleinGordon equation. By virtue of the equation of continuity of 


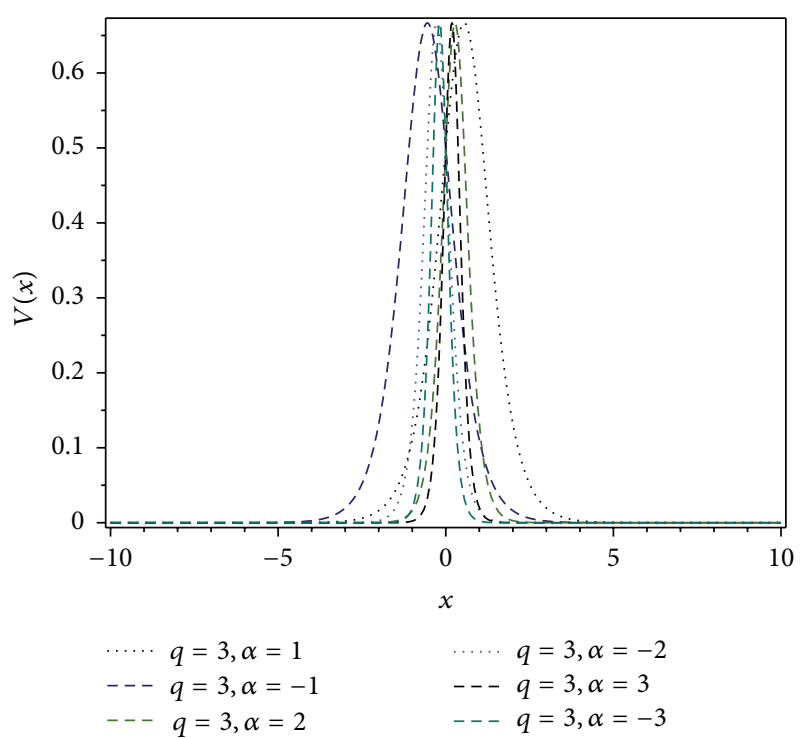

FIgURE 2: The plot of the qHPT potential as a function of position with $q=3, \alpha=1,2$, and 3 for $x<0$ and $x>0$, respectively.

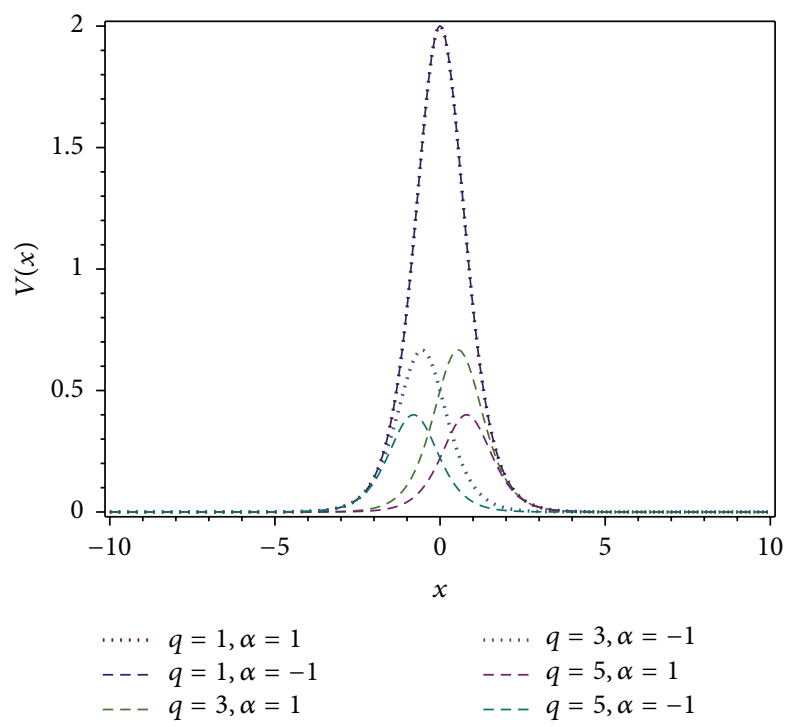

FIGURE 3: The plot of the qHPT potential as a function of position with $q=1,3$, and 5 for $x<0$ and $x>0$, respectively.

the wave function and the asymptotic properties of the solutions of the wave function, we calculate in detail the reflection and transmission coefficients and bound state solution by the vanishes of the determinant of the coefficients of the wave functions for the qHPT potential. This study can find many applications of physics especially in the interaction of nuclei in nuclear physics.

\section{Conflict of Interests}

The authors declare that there is no conflict of interests regarding the publication of this paper.

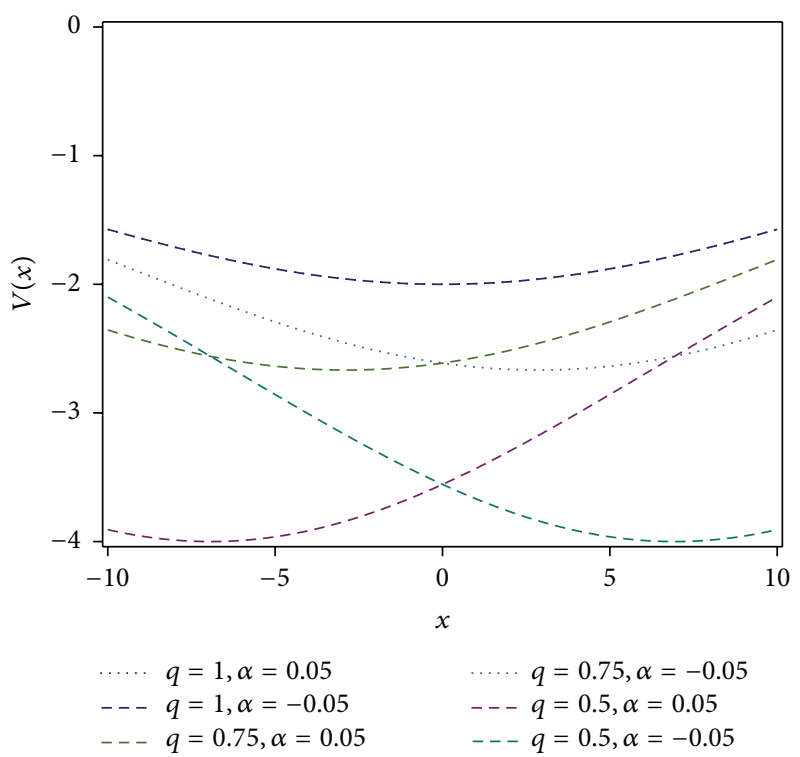

FIGURE 4: The plot of the qHPT potential as a function of position $x$ with $q=1,0.75$, and 0.5 and $\alpha=0.05$ for $x<0$ and $x>0$, respectively.

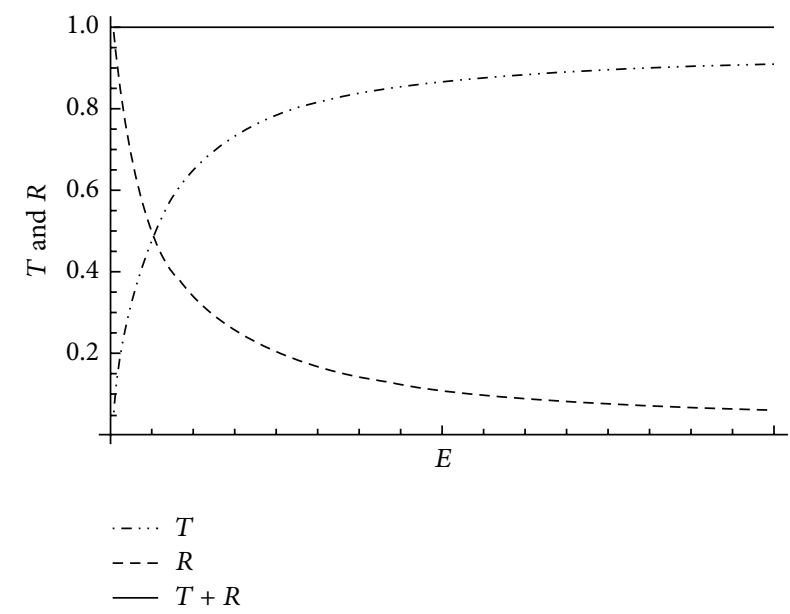

FIgURE 5: Transmission $(T)$ and reflection $(R)$ coefficients versus $E$ are depicted.

\section{Acknowledgment}

The authors are grateful for the right and faithful comments of the referees that improved the paper.

\section{References}

[1] N. Domhey, P. Kennedy, and A. Calogeracos, "Supercriticality and transmission resonances in the Dirac equation," Physical Review Letters, vol. 85, no. 9, pp. 1787-1790, 2000.

[2] V. M. Vilalba and C. Rojas, "Scattering of a Klein-Gordon particle by a Woods-Saxon potential," Physical Review A, vol. 71, no. 5, Article ID 052101, 4 pages, 2005.

[3] V. M. Vilalba and C. Rojas, "Scattering of a relativistic scalar particle by a cusp potential," Physics Letters A, vol. 362, no. 1, pp. 21-25, 2007. 
[4] H. Hassanabadi, E. Maghsoodi, N. Salehi, A. N. Ikot, and S. Zarrinkamar, "Scattering states of the dirac equation under asymmetric Hulthén potential," The European Physical Journal Plus, vol. 128, article 127, 2013.

[5] A. N. Ikot, H. Hassanabadi, E. Maghsoodi, and B. H. Yazarloo, "Bound and scattering states of modified Yukawa potential under relativistic spin and pseudospin symmetries with three tensor interactions," The European Physical Journal Plus, vol. 129, article 218, 2014.

[6] A. N. Ikot, B. H. Yazarloo, E. Maghsoodi, S. Zarrinkamar, and H. Hassanabadi, "Effects of tensors coupling to Dirac equation with shifted Hulthen potential via SUSYQM," Journal of the Association of Arab Universities for Basic and Applied Sciences, 2014.

[7] H. Hassanabadi, S. Zarrinkamar, and A. A. Rajabi, "A simple efficient methodology for Dirac equation in minimal length quantum mechanics," Physics Letters B, vol. 718, no. 3, pp. 1111$1113,2013$.

[8] H. Hassanabadi, S. Zarrinkamar, and E. Maghsoodi, "Minimal length Dirac equation revisited," The European Physical Journal Plus, vol. 128, article 25, 2013.

[9] H. Hassanabadi, Z. Molaee, and S. Zarrinkamar, "Noncommutative phase space schrödinger equation with minimal length," Advances in High Energy Physics, vol. 2014, Article ID 459345, 6 pages, 2014.

[10] A. Arda and R. Sever, "Effective-mass Klein-Gordon-Yukawa problem for bound and scattering states," Journal of Mathematical Physics, vol. 52, Article ID 092101, 2011.

[11] O. Aydoğdu, A. Arda, and R. Sever, "Effective-mass Dirac equation for Woods-Saxon potential: scattering, bound states, and resonances," Journal of Mathematical Physics, vol. 53, Article ID 042106, 2012.

[12] O. Aydoğdu, A. Arda, and R. Sever, "Scattering of a spinless particle by an asymmetric Hulthén potential within the effective mass formalism," Journal of Mathematical Physics, vol. 53, no. 10, Article ID 102111, 2012.

[13] C. Rojas and V. M. Vilalba, "Scattering of a Klein-Gordon particle by a Woods-Saxon potential," Physical Review A, vol. 71, no. 5, Article ID 052101, 4 pages, 2005.

[14] H. Hassanabadi, L. Lu, E. Maghsoodi, G. Liu, and S. Zarrinkamar, "Scattering of Klein-Gordon particles by a KINk-like potential," Annals of Physics, vol. 342, pp. 264-269, 2014.

[15] H. Hassanabadi, S. Zarrinkamar, and E. Maghsoodi, "Scattering states of Woods-Saxon interaction in minimal length quantum mechanics," Physics Letters B, vol. 718, no. 2, pp. 678-682, 2012.

[16] S. Alpdoğan, O. Aydoğdu, and A. Havare, "Relativistic spinless particles in the generalized asymmetric Woods-Saxon potential," Journal of Physics A: Mathematical and Theoretical, vol. 46, no. 1, Article ID 015301, 2013.

[17] A. Arda, O. Aydogdu, and R. Sever, "Scattering of the WoodsSaxon potential in the Schrödinger equation," Journal of Physics A: Mathematical and Theoretical, vol. 43, no. 42, Article ID 425204, 2010.

[18] S. Alpdoğan and H. Havare, "Dirac particle for the position dependent mass in the generalized asymmetric Woods-Saxon potential," Advances in High Energy Physics, vol. 2014, Article ID 973847, 10 pages, 2014.

[19] H. Yanar, A. Havare, and K. Sogut, "Scattering and bound states of Duffin-Kemmer-Petiau particles for q-parameter hyperbolic Pöschl-Teller potential," Advances in High Energy Physics, vol. 2014, Article ID 840907, 9 pages, 2014.
[20] A. Arai, "Exactly solvable supersymmetric quantum mechanics," Journal of Mathematical Analysis and Applications, vol. 158, no. 1, pp. 63-79, 1991.

[21] C. Grosche, "Path integral solutions for deformed Pöschl-Tellerlike and conditionally solvable potentials," Journal of Physics A: Mathematical and General, vol. 38, no. 13, pp. 2947-2958, 2005.

[22] L. D. Fadeev, "Properties of the $S$-matrix of the one-dimensional Schrödinger equation," Trudy Matematicheskogo Instituta imeni VA Steklova, vol. 73, pp. 314-336, 1964.

[23] P. Senn, "Threshold anomalies in one-dimensional scattering," The American Journal of Physics, vol. 56, no. 10, article 916, 1988.

[24] M. S. de Bianchi, "Levinson's theorem, zero-energy resonances, and time delay in one-dimensional scattering systems," Journal of Mathematical Physics, vol. 35, no. 6, pp. 2719-2733, 1994.

[25] D. Bohm, Quantum Mechanics, Printice Hall, Englewood Cliffs, NJ, USA, 1951.

[26] A. Calogeracos and N. Dombey, "Klein tunnelling and the Klein paradox," International Journal of Modern Physics A, vol. 14, no. 4, pp. 631-644, 1999.

[27] H. Hassanabadi, E. Maghsoodi, N. Salehi, A. N. Ikot, and S. Zarrinkamar, "Scattering states of the dirac equation under asymmetric Hulthén potential," The European Physical Journal Plus, vol. 128, article 127, 2013.

[28] M. Abramowitz and I. A. Stegun, Handbook of Mathematical Functions, Dover Publications, New York, NY, USA, 1970.

[29] N. Candemir and O. Bayrak, "Massive Dirac equation in asymmetric Hulthén potential," Journal of Mathematical Physics, vol. 54, Article ID 042104, 2013.

[30] O. Panella, S. Biondini, and A. Arda, "New exact solution of the one-dimensional Dirac equation for the Woods-Saxon potential within the effective mass case," Journal of Physics A: Mathematical and Theoretical, vol. 43, no. 32, Article ID 325302, 2010.

[31] V. M. Villalba and C. Rojas, "Bound states of the Klein-Gordon equation in the presence of short range potentials," International Journal of Modern Physics A, vol. 21, no. 2, pp. 313-326, 2006.

[32] Z. Molaee, H. Hassanabadi, and S. Zarrinkamar, "Scattering states of Schrödinger equation under the modified cusp potential," Communications in Theoretical Physics, vol. 60, no. 1, pp. 25-27, 2013. 

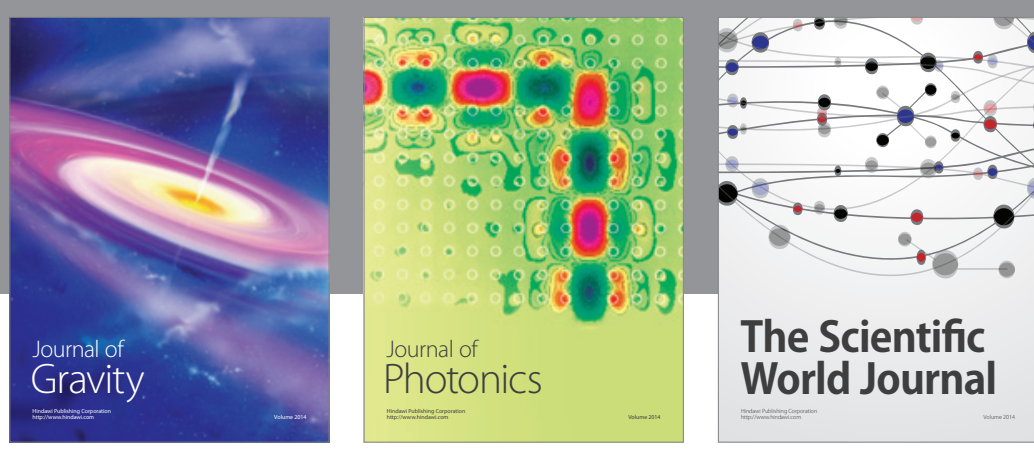

The Scientific World Journal
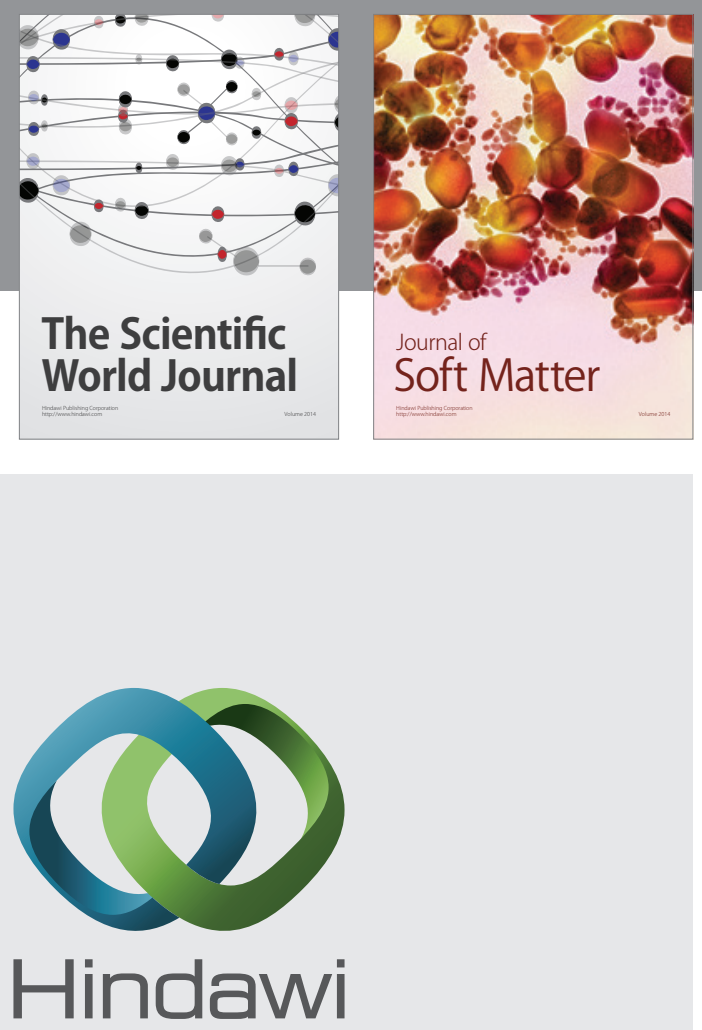

Submit your manuscripts at

http://www.hindawi.com

nternational Journal of

Statistical Mechanics
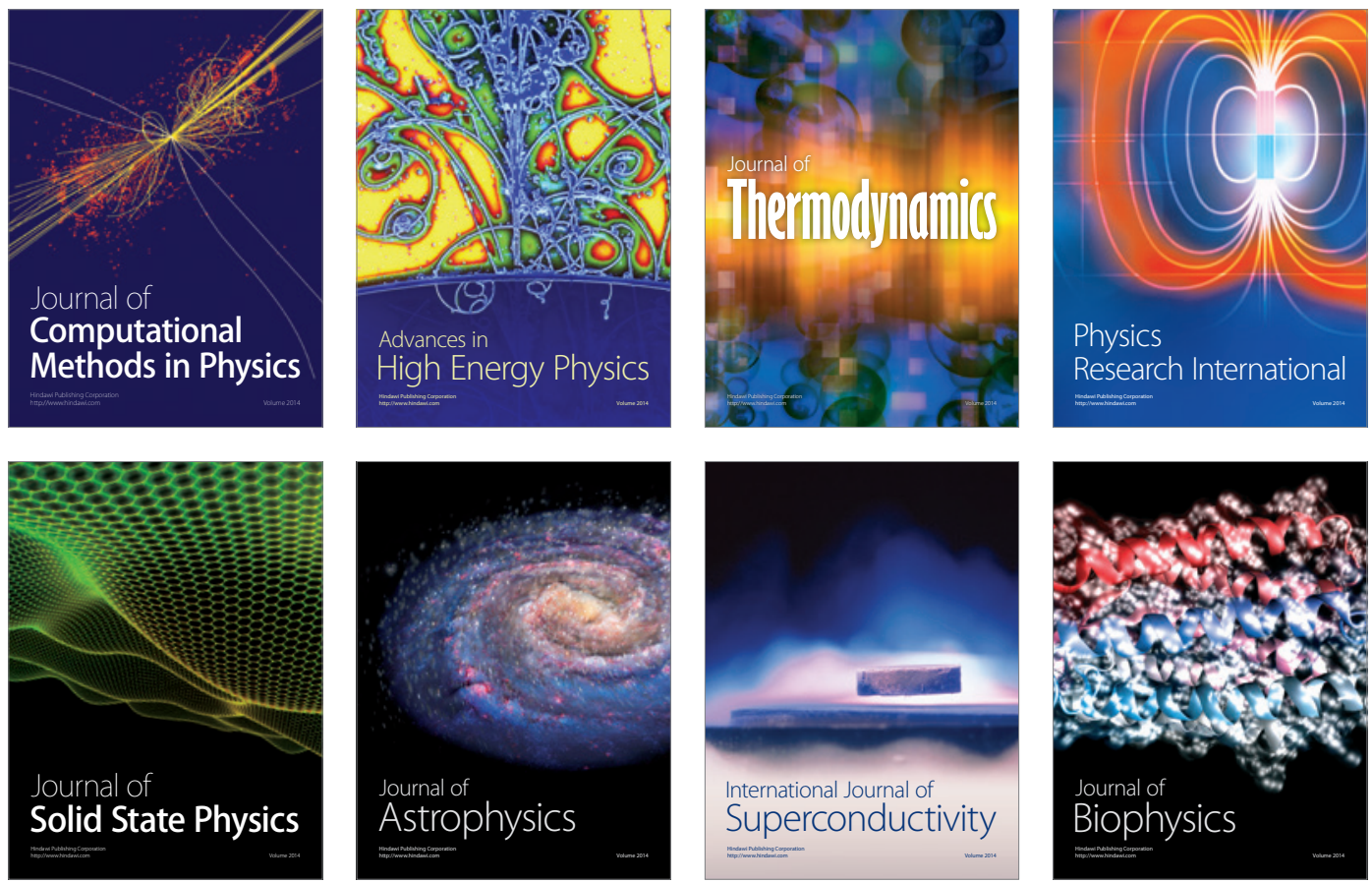
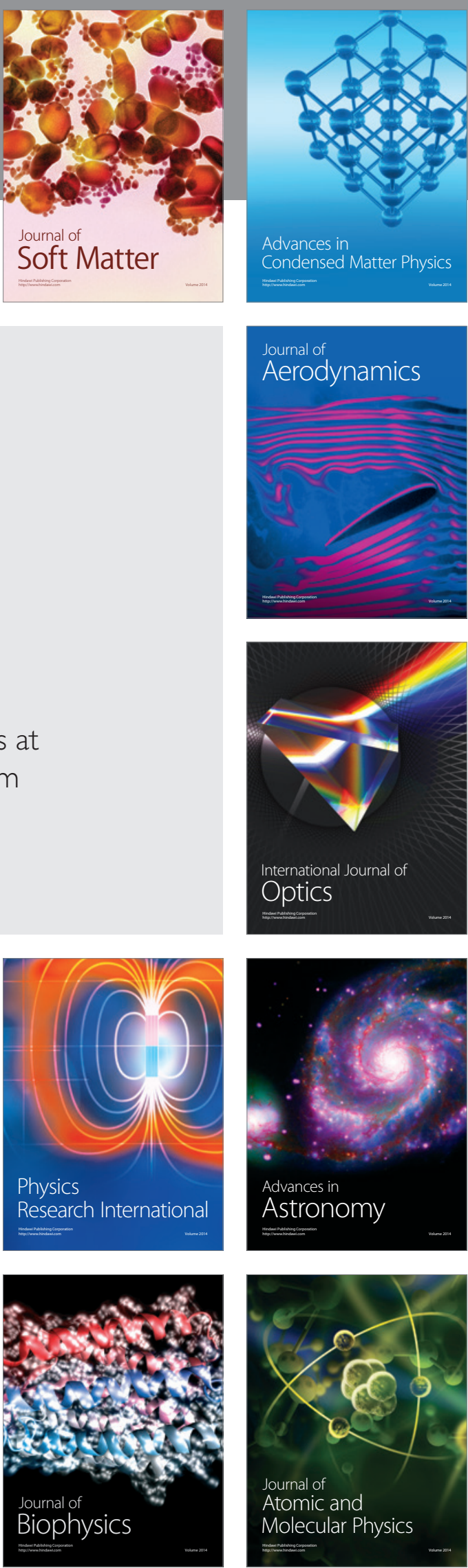